\title{
LADINIA LINGUISTICA IN UNA MONUMENTALE OPERA: ATLANTE LINGUISTICO DEL LADINO DOLOMITICO E DEI DIALETTI LIMITROFI - ALD-1, DR. LUDWIG REICHERT VERLAG, WIESBADEN 1998.
}

L'apparizione dell'atlante linguistico di un territorio romanzo va salutata con gioia, come una festa della ricerca scientifica in tale settore. $E$ il sentimento di gioia ci pervade quando sfogliamo i primi quattro volumi, in folio, dell'ALD. Non solo per la mole e l'ampia concezione dell'opera, ma altrettanto e più ancora per il ricco materiale che l'ALD offre. A tutta l'équipe scientifica guidata dal rinomato romanista salisburghese prof. Hans Goebl che assieme a Lois Craffonara è anche ideatore dell'opera, nonché ai collaboratori e all'editore vadano i nostri sinceri ringraziamenti. Ci sia permesso di aggiungere che la nostra rivista si pregia d'aver potuto ospitare alcuni studi scientifici del prof. Goebl, come anche del suo stretto collaboratore prof. Roland Bauer.

Ai primi quattro volumi con i 217 punti esplorati e le 884 cartine seguono tre volumi con gli indici: uno alfabetico, uno inverso e uno etimologico, quest'ultimo sulla base del vocabolo, stimulo, in italiano, nel questionario dell'Atlante sovente inserito in un sintagma $o$ in una mezza frase, il fatto che rende la risposta più veritiera. L'ALD-I ha sfruttato, così pare, tutti gli strumenti della moderna tecnologia: il materiale raccolto è disponibile anche in CD-ROM. Inoltre, i materiali raccolti nei punti esplorati nella Ladinia dolomitica (punti 61 - 101) sono accessibili anche nella versione fonica: una vera novità, anche se si pensa alla Carta dei dialetti italiani di Oronzo Parlangèli.

Nello stesso tempo, oltre all'entusiasmo per l'opera compiuta, l'apparizione di un tale lavoro può legittimamente suscitare anche la domanda sulla ragione di un'impresa di tale tipo e di tali proporzioni. Oggidi, tutti (o quasi) sappiamo leggere e scrivere e già per il passato è stato detto che, essendosi verificata, pare, la scolarizzazione di tutta la popolazione, le divergenze in una stessa lingua, anche nelle questioni riguardanti la pronuncia e la grafia, si erano attenuate. Si riconosce per la normalizzazione della scrittura l'importanza della stampa, cinquecento anni fa; un po' così, l'immagine fonica di una lingua avrebbe subito una sensibile semplificazione. In misura minore questo fatto sarà dovuto all'influenza dell'ascolto della radio; molto più radicale e decisivo sarà l'ascolto della catena sonora della TV. Se non i partecipanti occasionali, almeno i collaboratori professionali, annunciatori e presentatori offrono con la lingua standard anche la pronuncia standardizzata. Poco meno di cent'anni fa appariva l'ALF, l'Atlas Linguistique de la France, e già in quel tempo fu avanzata l'idea che, per la Francia, quell'atlante dovesse essere l'ultima opera del genere. Le parlate regionali 
avrebbero dovuto scomparire davanti alla più o meno unitaria lingua nazionale. Ciò non si è verificato: al posto dei dialetti che scomparivano, a condizione che davvero stessero per scomparire, si faceva forte lo standard regionale che segue, sì, la norma della lingua scritta, letteraria, ma dimostra nello stesso tempo, e soprattutto nella pronuncia, tratti peculiari. Vorremmo però mettere in rilievo che il materiale raccolto da un atlante linguistico è importante non solo perché permette di comparare le varie realizzazioni dialettali con lo stato nella lingua letteraria, ma anche, e forse di più, con la situazione linguistica riscontrata nelle parlate vicine, limitrofi. E l'ALD è prezioso soprattutto sotto questo aspetto, trascurato per lo più quando si mettono a confronto solo lingue letterarie.

La pubblicazione di un atlante linguistico non è, nei nostri tempi, con tutto il progresso tecnologico un'azione superflua, sorpassata. E meno che mai lo è in una situazione in cui si trova, linguisticamente, la parte del territorio alpino che è stata l'oggetto essenziale della ricerca per l'ALD.

Le presenti note prendono in esame ovviamente solo il materiale dei volumi pubblicati che rappresentano la 1.a parte dell'intero lavoro. Seguirà la seconda parte che potrà essere da un lato ancora più interessante, giacché dovrebbe renderci palese gli aspetti morfosintattici e semantici, lessicali. La prima parte dell'opera, l'ALD-I, è destinata, così spiega nell'Introduzione il direttore della ricerca, a presentare la veste fonica e con questa il panorama fonetico. E' vero; il materiale raccolto, tuttavia, rende, benché non sistematicamente, ampie informazioni su alcuni problemi morfologici: formazione del femminile e del plurale dei sostantivi e aggettivi, varietà delle forme verbali. E benché l'opera si dichiari, sempre nell'Introduzione, strumento per constatare l'immagine fonica, servirà non poco anche per il lato semantico: per lo stesso concetto presenta vari lessemi, il che in un territorio in buona parte di montagna non deve sorprendere; al contrario. Per ciò, tanto più impazienti aspettiamo l'apparizione della seconda parte dell'Atlante.

Siamo convinti, dunque, che la raccolta del materiale che precede la pubblicazione di un atlante linguistico sia ancora sempre di grande importanza, così come lo era stata nei primi lavori del genere, vale a dire, alla fine dell'Ottocento; siamo inoltre dell'opinione che la lingua letteraria con la sua norma rimanga pur sempre un'astrazione, che il materiale raccolto per un atlante linguistico sia autentico, genuino, che rifletta fedelmente lo stato di lingua di un dato periodo, quello attuale, e infine che nello stesso tempo col dato annotato possa informarci su vari cambiamenti avvenuti nel corso della storia di una lingua.

Il territorio che l'ALD abbraccia è riccamente intrecciato. Si sa che nella cerchia romanistica non si è giunti all'accordo riguardo alla classificazione linguistica delle parti del territorio. Anzi, la denominazione stessa è la prova esteriore, superficiale dell' imbarazzo in cui ci si trova trattando le parlate romanze alpine: ci siamo serviti, appunto per toglierci dall'imbarazzo o almeno per attenuarlo, della terminologia di Gamillscheg. Dobbiamo felicitarci con la direzione dell'ALD: nel testo ladino e italiano delle 
parti preliminari il termine usato è quello del ladino, mentre nel testo steso in tedesco incontriamo il Rätoromanisch. E' eliminato, con tutta l'eleganza, il disagio che avrebbe potuto complicare le cose sin dall'inizio, con la denominazione. Del resto, seguendo il saggio modello della Confederatio Helvetica, l'Atlante ricorre al latino quando la dizione nelle tre lingue usate nei capitoli introduttivi dovesse rendere le informazioni, le didascalie troppo impacciate. Un atlante linguistico, poi, ha lo scopo di offrire del materiale raccolto onestamente, con tutta l'acribia scientifica, e con questo rende possibile risolvere vari problemi linguistici; non cerca di complicarli e solo più tardi, valutando questo materiale, può sorgere una disputa.

I punti che possono. al contrario, essere discussi sono, così pensiamo, i seguenti: il territorio esplorato, la composizione del questionario, eventualmente anche la grafia.

Quanto al territorio esplorato: 1'ALD-I abbraccia il territorio del ladino dolomitico, vale a dire del ladino stricto sensu. Purtuttavia, l'esplorazione non si è limitata al ladino che siamo soliti chiamare il ladino dolomitico (Val Badia, Val Gardena, Val di Fas$\mathrm{sa}$, Val di Fiemme), alla sola Ladinia dolomitica dunque. Sono presenti, del territorio considerato ladino (largo sensu, questa volta), anche parte del retoromancio (la bassa Engadina e la Val Monastero, geograficamente parte della Svizzera sud-orientale) e parte del friulano occidentale. Inoltre, all'infuori dell'area strettamente ladina, troviamo esplorate anche località della Lombardia orientale, tutto il Trentino e parte del Veneto centrale e settentrionale. In sostanza, le anfizone ascoliane (pensiamo all'AGI I, par. 4. Ladino e Veneto). In tutto sono stati esplorati 217 punti, per lo più da due esploratori, e il materiale raccolto è sistemato su 884 cartine, alcune delle quali "doppie", come già detto maschile/femminile, singolare/plurale. Il direttore, il quale, come dice il frontespizio, opus omne curavit, spiega nell'Introduzione che "l'obiettivo prioritario dell'ALD-I è /.../ fornire al lettore interessato una pubblicazione solidamente elaborata, di facile consultazione e dotata di strumenti più attuali." Vorrei menzionare per inciso un piccolo dettaglio per mettere in rilievo il costante e solido lavoro dell'équipe scientifica. Le cartine che si trovano nell'ALD-I sono, geograficamente, accessibili e facilmente consultabili, giacché sul loro sfondo appare il territorio con le principali località, i fiumi, le vallate, mentre i punti esplorati appaiono, ovviamente, cifrati; lo sfondo geografico aiuta non poco chi consulta la cartina, se non è proprio familiare di quei luoghi, per orientarsi meglio.

Detta amplificazione geografico-linguistica non incontrerà, forse, il consenso in tutti. Però, l'AIS, ad esempio, che pur rimane il modello per ogni atlante linguistico, è stato dichiarato nel suo titolo "dell'Italia e della Svizzera meridionale". Pare che il titolo sia stato scelto allo scopo di evitare polemiche linguistiche e, per quei tempi, forse anche politiche. Nella sua Grammatica storica della lingua italiana e dei suoi dialetti (il titolo è significativo), Gerhard Rohlfs, egli stesso esploratore dell'AIS per l'Italia meridionale, evita di citare esempi sardi, friulani, francoprovenzali. Però, per l'ALD ben volentieri accettiamo tale amplificazione: essa ci dà l'opportunità di comparare la Ladinia linguistica con almeno una parte del territorio ladino, largo sensu, la bassa 
Engadina e il Friuli occidentale. Inoltre, è prezioso il paragone con la situazione linguistica nel Trentino e nella Lombardia orientale. La conoscenza dei dialetti veneti, poi, è di particolare importanza; non è nemmeno necessario richiamare l'attenzione sulle ben note idee di Carlo Battisti riguardo all'attribuzione linguistica dei tre tronconi ladini di Ascoli ai dialetti dell'Italia settentrionale.

Il nostro interesse è rivolto, ovviamente, in maniera particolare alla situazione linguistica della Ladinia. Si precisa, ripetiamo, nell'Introduzione dell'ALD-I che viene concesso ampio spazio ai fenomeni fonetici. Vediamo, infatti, che nelle risposte dei singoli informatori si possono constatare le seguenti innovazioni fonetiche rispetto allo stato della stessa unità linguistica in latino e paragonare gli esiti in varie parlate.

Vocali

a) vocali toniche: la A lunga latina si palatalizza, ćanté, l pré, la sel, anche in sillaba chiusa, la pert, mentre rimane invariata in frl. e in eng., come nel resto dei territori esplorati (Veneto, Lombardia); la U lunga latina si palatalizza in lad. e in eng., madür, krü contro il frl. madûr, krût/kruda; la O lunga latina si chiude in lad. e in eng. kruš, dolur, mentre rimane invariata o dittonga in frl. krôš, dolôr/dolour; in sillaba chiusa le vocali mediane, brevi in lat., dittongano solo in frl. al muart 'morde', muart/muarta 'morto, -a'; così fiešta, mentre troviamo in lad. e eng. fešta;

b) dittonghi: il dittongo latino AU si chiude dappertutto, anche nel frl. occidentale; non così nel frl. centrale;

c) vocali atone finali: cadono in tutte e tre le sezioni; la sincope è scarsa, dodǐs, quasi inesistente in frl., cfr. FEMINA, frl. femena contro fena in lad. e eng.

Consonanti

a) Per la sorte delle consonanti è ovvio che, essendo la zona esplorata l'area della Romania occidentale, la sonorizzazione delle occlusive latine intervocaliche non sorprende e nemmeno la lenizione o la fusione totale della consonante nell'entourage vocalico: VITELLU lad. videl, it. strega è stria.

b) La palatalizzazione delle consonanti fa un capitolo a sé: il materiale raccolto offre quella panromanza, ossia delle occlusive latine davanti a una vocale palatale in iato e quella delle occlusive velari davanti alle vocali palatali; ne deriva la palatalizzazione oppure la sibilantizzazione: ćink/sink. Sempre problematica è l'interpretazione della sorte delle velari davanti alla vocale -A. Il territorio esplorato conferma la palatalizzazione nelle tre aree "ladine" (sit venia verbo) e la conservazione del timbro velare altrove: lad. ial, iarina/đarina, iama e così in eng. e frl. yal, dial; yalina, đialina; đoma/iome, điamba contro a gal ecc. in veneto e altrove con la velare conservata.

c) Attraente per la sua varietà è la sorte dei gruppi consonantici con la liquida $-l$. La liquida si conserva in tutti e tre i tronconi ladini e i gruppi latini PL-, BL-, FL- sono stati ereditati tali e quali. Per il gruppo latino $\mathrm{KL}$, invece, il ladino dolomitico offre 
come esiti di *MASCLU, *GENUCLU, AURICLA, CLARU, it. chioccia: mandl ( $n$ dal tedesco ?), ženedl, oredla, tler, tloća.

d) Le affricate si semplificano, vale a dire, perdono l'elemento dentale, riflettendo un fenomeno fonetico generale; detsembr, certo, è voce dotta.

e) Il rotacismo è tipico solo dell'area ladina dolomitica: mora, murin, firé, štera trovano altrove esiti concordi agli it. mola, mulino, filare, stella. Qualche discordanza si trova anche nella Ladinia stessa: la Val Badia offre škora, la Val Gardena škola.

f) In seguito alla sincope avvenuta, si verifica in lad. e in eng. l'inserto di una consonante omorganica per evitare il gruppo consonantico insolito: GENEREM žendre, *CINERE čendra, čeindr. In friulano la sincope è rara e perciò il problema non si pone: ginar, ceniza/siniza.

g) Il gruppo consonantico -KT- subisce l'assimilazione dell'elemento velare a quello seguente, dentale, il che è tipico della lingua italiana, e poi la semplificazione della doppia: *LACTE lat. Il frl. semplifica anche il gruppo -GW-: LINGUA lenga.

h) E' sorprendente che il gruppo consonantico -MB-, conservatosi in altre-parti del territorio esplorato, dimostri solo in ladino e parzialmente in engadino, in Val di Fassa e in Livinallongo l'assimilazione progressiva e poi la semplificazione della consonante doppia: it. gamba čama/čoma/yome; lo stesso fenomeno si trova nella forma femminile dell'aggettivo gran, grana e, accanto a sponda (cartina con stimulo "riva") come špona. Il fenomeno è sorprendente perché, attribuito all'influenza del sostrato osco-umbro, pare riservato all'Italia centro-meridionale.

i) La $-r$ finale è conservata in engadinese, mentre è caduta in ladino e in friulano.

E' spiegato nell'Introduzione che nell'ALD-I sono evidenziati solamente alcuni fenomeni morfologici e morfosintattici. E' vero, sono pochi; però, quello che si può ricavarne è importante. Per il sostantivo e l'aggettivo troviamo infatti presentata, giacché si contrappone a quella del maschile, la forma del femminile: vert, verta; poi, quella del plurale contrapposta alla forma del singolare. Il dato interessa non solo per gli eventuali cambiamenti fonetici; il materiale raccolto conferma che il friulano e, a volte, anche il ladino possiedono, accanto a quelli sigmatici, anche plurali asigmatici, come ćavai, fasoi.

Particolarmente preziose, poi, sono le informazioni sul genere degli alberi da frutto: decaduto il sistema logico e solido del latino, le lingue romanze cercano di ristabilire in qualche modo l'opposizione "albero (da frutto) - frutto". Così troviamo per il concetto di albero: dol. leñ de keršes, leñ de per e nell'eng. e frl. per il primo, rispettivamente, ćarě̌er e čeriezar.

All'infuori di questa specifica categoria si constata che il genere del sostantivo latino è per lo più conservato, con qualche rara eccezione, come eng. il man, i mans oppure lad. la lüme. DIES deve essere stato in quest'area di genere femminile, o almeno anche femminile, giacché troviamo, sì, il lunedi, ma eng. la dumenđa e lad. la dumenia/la domáña, il che, del resto, non discorda con il passo biblico di UNA AUTEM SABBATI, Joan., XX, 1. 
Il morfema $-a$, la caratteristica dell'italiano per la formazione del plurale di una classe di sostantivi maschili (Il plurale italiano in -a: un duale mancato?, per sfruttare il titolo di uno studio di Robert Jr. Hall, Italica 33, del 1956), non è conosciuto nel mondo ladino; non lo è nemmeno nei dialetti dell'Italia settentrionale: le ginocchia sono lad. $i$ zenedl, eng. šnoels, frl. đenoi, i zenoći. Col morfema - a si trova solo la leña 'il legname per ardere', poche volte le leñe.

E' utile trovare accanto al sostantivo il rispettivo articolo determinativo, sia per il singolare che per il plurale.

Per il verbo c'è da notare che sono, saggiamente, indicate varie forme verbali: l'infinito, il presente (1.a pers.), il participio passato. Dalle forme personali risulta che in tutta questa zona il verbo richiede la presenza del pronome personale atono e in alcune zone, in frl. ad es., addirittura, il pronome personale in forma tonica e atona: lui al da, lui al diš. Il fenomeno è conosciuto, benché limitato ad alcune persone, anche in lombardo e in veneto.

Per il lessico ripetiamo che l'ALD-I è destinato alla descrizione fonica; aspettiamo dunque una messe lessicale abbondante nella seconda parte dell'opera, ossia una nomenclatura variopinta, giacché ci troviamo, almeno per la parte ladina (lato sensu), nell'area alpina e prealpina. Inoltre, si sa che soprattutto in campagna i termini generici non sono troppo familiari; è giusto quello che i redattori hanno messo nella "legenda" alla cartina pianta: "Essendo estraneo a molti dialetti un concetto generico per qualsiasi vegetale erbaceo, arbustivo o arboreo." Il parlato vuole essere concreto.

Alcune cartine attirano il nostro interesse in modo particolare:

a) le risposte alla domanda in cui è incluso il termine cucchiaio informano che sedon germanico, gotico è davvero il termine che accomuna i tre tronconi della (presunta e discussa) unità linguistica del ladino, mentre in tutte le altre aree è conosciuto solo il vocabolo di origine greca, quasi panromanzo;

b) la cartina con il lemma calzoni rileva la vitalità della parola celtica braca, sparita, o quasi, nel paese d'origine, ma conservata nell'Italia settentrionale, poi, anche in un dialetto sloveno occidentale, il resiano (brage), e persino nella lontana Dacia: il rumeno tutt'ora conosce $s$ 'îmbrăca, îmbrăcăminte 'vestirsi, vestimento';

c) l'ALD-I mette in rilievo che i termini religiosi sono abbastanza unitari e che sono decisamente degli italianismi: epifania. Ciò vale in generale per i termini dotti. Quando invece un simile termine diventa patrimonio della lingua parlata, la scelta è più libera: diavol, diaul, daul si trovano lessicalmente in contrasto con il termine ladino le malan;

d) per l'apporto delle lingue attigue si constata un numero piuttosto limitato di vocaboli di provenienza tedesca, ad es., pfefer, broast/prust, stop per 'pepe', 'petto', 'polvere', rispettivamente. Più frequenti sembrano essere i tedeschismi in eng.: šnaidar, saide, ecc. per 'sarto', 'seta'. 
$\mathrm{E}$, siccome scrivo queste righe nell' area linguisticamente slovena, non posso non notare cosse 'cesta' e sespa 'susina' di provenienza slovena, termini che appaiono com'è prevedibile solo nell'area friulana.

Per quanto riguarda il lato tecnico non abbiamo nessun rimprovero da formulare, né consigli da dare: la veste tipografica è impeccabile, errori tipografici non ce ne sono, malgrado le somme difficoltà nel rendere, graficamente, i vari suoni che gli esploratori hanno captato nelle risposte dei loro informatori. E' giusto che questa ricchezza fonica sia stata un po' affievolita nei volumi aggiunti con la semplificazione della grafia, criterio seguito anche dallo scrivente.

Siamo dunque in presenza di un lavoro monumentale, accuratamente concepito, dettagliatamente preparato e eseguito con massima sollecitudine. Il risultato è la prima parte dell'ALD, dove troviamo raccolti in rassegna tutti gli importanti fenomeni fonetici, e non solo questi, che risultano dal paragone con il lemma italiano preposto ad ogni cartina. Più prezioso ancora è il possibile paragone tra le zone attigue: il materiale raccolto, genuino, permette di riconoscere le isoglosse fonetiche che accomunano $i$ tre tronconi della Ladinia (sempre nel senso ascoliano), spinge addirittura a valutare le convergenze e le divergenze linguistiche di detti territori con le vicine e attigue aree linguistiche dell' italiano settentrionale.

Se gli autori, gli esploratori e i collaboratori hanno dedicato quindici anni alla preparazione del loro atlante, come viene spiegato nella prefazione, possono essere certi che $\mathrm{i}$ risultati raggiunti, vale a dire il materiale raccolto e messo a disposizione degli studiosi interessati, saranno per molte generazioni, compresa la nostra e quelle a venire, la fonte principale per ogni valutazione dei problemi toccanti i fenomeni linguistici del territorio ladino $\mathrm{e}$ anche più genericamente romanzo. Per una migliore conoscenza della problematica ladina vorremmo augurarci che la seconda parte del lavoro promessa veda la luce del giorno in un lasso di tempo ragionevole. Siamo in molti che aspettiamo la sua apparizione, non oso dire impazientemente, giacché tale espressione non si addice al meticoloso lavoro di ogni impresa di alto valore scientifico e meno che mai a un lavoro di così ampio respiro, ma comunque in un futuro non troppo lontano.

Povzetek

\section{JEZIKOVNI ATLAS DOLOMITSKE LADINŠČINE}

Prispevek tehta in ocenjuje gradivo, ki je zbrano v jezikovnem atlasu Ladinije (ALD-I), se pravi atlasu romanskih govorov $v$ italijanskih Dolomitih. Zanimanje in različni pogledi na ta romanski svet so starega datuma, začenši z znamenito študijo vélikega italijanskega jezikoslovca G. I. Ascolija $\mathrm{z}$ naslovom Saggi ladini, objavljeno v reviji Archivio Glottologico Italiano, I, 1873, kjer so furlanščina, romanski govori v Dolomitih, (reto)romanski govori v švicarskem kantonu Graubündnu obravnavani kot deli neke jezikovne enote. Čisto drugačen je bil pogled Carla Battistija (vrsta objav o tej témi od leta 1910 dalje), ki ima vse tri "ladinske" dele za podaljške severnoitalijanskih narečij.

Atlas dolomitske ladinščine, delo močne znanstvene ekipe pod vodstvom Hansa Goebla $z$ uni- 
verze v Salzburgu, je rezultat znanstvenih raziskav zadnjih petnajstih let. Prvi del, ki je zdaj objavljen, je posvečen zlasti raziskavi glasoslovja. Posebej je dragoceno, da se raziskovanje na terenu ni omejilo samo na romanske govore v Dolomitih, ampak je bilo razširjeno na stična ladinska ozemlja (v slovenščini, po nemškem vzorcu, raje uporabljamo términ "retoromanski"), na švicarski Engadin in na zahodno Furlanijo, a tudi na severnoitalijanski narečji, severnobeneško in vzhodno lombardsko. $S$ tem je omogočena primerjava, ugotavljanje skupnih fonetičnih inovacij glede na latinščino, omogočena pa je tudi primerjava med posameznimi govori tega področja, ne da bi se pri tem vsiljevalo kakršno koli vnaprejšnjo klasifikacijo.

Nabrano in zapisano gradivo izvira iz anket na 217 točkah in je zaobseženo na 884 kartah, kar pomeni, da je predstavljeno visoko število izrazov za teh 884 gesel. Ker gre za geografsko omejeno ozemlje, je mreža gosta, raziskane točke so si oddaljene nekako $10 \mathrm{~km}$. Rezultati anket so zbrani $\mathrm{v} 4$ zvezkih velikega formata, $\mathrm{k}$ temu pa je treba dodati še tri spremljevalne zvezke (gesla po abecedi, obrnjeni slovar, etimološki slovar gesel).

Atlas je zelo moderno zastavljen: dostopen je tudi na CD-ROMu, Poleg tega pa je anketiranje osrednjega dela ozemlja, torej tiste prave Ladinie (Val Badia, Val Gardena, Val di Fassa, Val di Fiemme) posneto tudi na slušni disketi, kar je seveda velika novost.

Epohalno delo široke zasnove, prvo svoje vrste za ladinščino, je zvest prikaz sedanjega stanja romanskih govorov v Dolomitih. Prvi del je res posvečen predstavitvi glasovnih pojavov, vendar pa najdemo marsikaj dragocenega tudi za oblike, tako pri samostalniku razlikovanje med oblikama za moški in ženski spol, za ednino in množino, ali pri glagolu za osebne in neosebne oblike. Zbrano gradivo daje zanimiv, čeprav nesistematičen pregled za besedje, kakor ga je raziskovalec $\vee$ kraju ankete pač našel in zapisal: za razliko od slovarja, kjer se navajajo različni pomeni in rabe enega in istega izraza, je naloga jezikovnega atlasa ravno $v$ tem, da $z a$ isti pojem navede nabrane izraze; v gorskem svetu je raznolikost sama po sebi razumljiva. V tej smeri bo seveda drugi del Atlasa še zanimivejši. 